\title{
HARMONIC MAPS WITH PRESCRIBED SINGULARITIES INTO HADAMARD MANIFOLDS
}

\author{
Gilbert Weinstein
}

\begin{abstract}
Let $M$ a Riemannian manifold of dimension $m \geqslant 3$, let $\Sigma$ be a closed smooth submanifold of $M$ of co-dimension at least 2 , and let $H$ be a Hadamard manifold with pinched sectional curvatures. We prove the existence and uniqueness of harmonic maps $\varphi: M \backslash \Sigma \rightarrow H$ with prescribed singularities along $\Sigma$. When $M=\mathbb{R}^{3}$, and $H=H_{\mathrm{c}}^{k}$, the complex hyperbolic space, this result has applications to the problem of multiple co-axially rotating black holes in general relativity.
\end{abstract}

\section{Introduction}

In [We3, We4], we showed how the Einstein/Abelian-Yang-Mills equations, under the assumptions of stationarity, axial symmetry, asymptotic flatness, together with regularity and non-degeneracy conditions, are equivalent to a harmonic map problem with prescribed singularities into the complex hyperbolic space $H_{\mathbb{C}}^{k}=S U(1, k) / S(U(1) \times U(k))$. We then used a variational method to prove the existence of such harmonic maps with prescribed singularities into any hyperbolic space, $H_{\mathbb{K}}^{k}$ where $\mathbb{K}$ is either the real numbers $\mathbb{R}$, the complex numbers $\mathbb{C}$, or the quaternions $\mathbb{H}$.

In this paper, we generalize this result, and prove the existence and uniqueness of such maps for any target $H$ which is a Hadamard manifold with pinched sectional curvatures $-b^{2} \leqslant \kappa \leqslant-a^{2}<0$. The proof we present here is also considerably simpler than in these earlier papers.

In order to state our result, we need to introduce a few notions. Let $(M, g)$ and $(N, h)$ be Riemannian manifolds and let $\varphi: M \rightarrow N$ be a map of class $C^{1}$. The energy density of $\varphi$ is the length squared of the differential of $\varphi$ :

$$
|d \varphi|^{2}=\sum_{\substack{i, j=1, \ldots, n \\ \mu, \nu=1, \ldots, m}} h_{i j} g^{\mu \nu} \partial_{\mu} \varphi^{i} \partial_{\nu} \varphi^{j}
$$

Received July 11, 1996.

1991 Mathematics Subject Classification. 58E20, Secondary 83C57.

Key words and phrases. Harmonic maps, singularities, Hadamard manifolds, rotating black holes.

This work was supported in part by NSF Grant DMS-9404523. 
where on the right-hand side we have used local coordinates on both $(M, g)$, and $(N, h)$. The energy $E_{\Omega}(\varphi)$ of $\varphi$ over $\Omega \subset M$ is then the integral of the energy density:

$$
E_{\Omega}(\varphi)=\int_{\Omega}|d \varphi|^{2}
$$

A map $\varphi$ is harmonic if for each $\Omega \subset \subset M, \varphi$ is a critical point of $E_{\Omega}(\varphi)$. Here, we have written $\Omega \subset \subset M$ to mean $\Omega$ is open and $\bar{\Omega}$ is compact in $M$. If $\varphi$ is harmonic, then it satisfies the following elliptic system of partial differential equations:

$$
\Delta_{g} \varphi^{i}+\sum_{\substack{j, k=1, \ldots, n \\ \mu, \nu=1, \ldots, m}} \Gamma_{j k}^{i}(\varphi) \partial_{\mu} \varphi^{j} \partial_{\nu} \varphi^{k} g^{\mu \nu}=0,
$$

where $\Delta_{g}$ denotes the Laplacian with respect to the metric $g$, and $\Gamma_{j k}^{i}$ are the Christoffel symbols of the metric $h$. The right-hand side of (1) are the components in local coordinates of a section of the pull-back bundle $\varphi^{-1} T N$, which is called the tension field of $\varphi$, and which we denote by $\tau(\varphi)$. Thus, the function $|\tau(\varphi)|=\sqrt{h(\tau(\varphi), \tau(\varphi))}$ on $M$ is a measure of how much $\varphi$ fails to be harmonic.

Harmonic maps have been studied extensively. When the target space is nonpositively curved, finite energy harmonic maps are well understood; see [ES, H, SU1, SU2]. In particular, if $\varphi: M \rightarrow N$ is any finite energy harmonic map on a ball $B \subset M$, and $N$ is negatively curved, then $\varphi$ is smooth, i.e., of class $C^{\infty}$. Furthermore, if $M$ is a compact manifold with non-empty boundary, $N$ is simply connected, negatively curved, and convex, and $\psi: M \rightarrow N$ is a smooth map, then there is a harmonic map $\varphi: M \rightarrow N$ such that $\varphi=\psi$ on $\partial M$, see [H, SU2]. In this paper, we study the Dirichlet problem for harmonic maps with prescribed singularities along a submanifold $\Sigma$ of $M$ of co-dimension $\geqslant 2$.

Let $\Sigma$ be a compact smooth submanifold of $M$ of co-dimension at least 2 , possibly with $\partial \Sigma \neq \emptyset$. By taking a positive distribution on $\Sigma$, and convoluting with the fundamental solution on $M$, we obtain a harmonic function $u$ on $M \backslash \Sigma$ which tends to $+\infty$ on $\Sigma$. Let $H$ be a Hadamard manifold with pinched sectional curvatures $-b^{2} \leqslant \kappa \leqslant-a^{2}<0$, and let $\gamma$ be a geodesic of $H$. Then clearly $\varphi=\gamma \circ u$ is a harmonic map whose image lies entirely in the geodesic $\gamma$, and $\varphi(x)$ approaches the ideal point $\gamma(+\infty) \in \partial H$ as $x \rightarrow \Sigma$. We will call any harmonic map $\varphi$ which satisfies these two conditions a $\Sigma$-singular map into $\gamma$. Singular maps of this type will be used to prescribe the singularities along $\Sigma$. To this end we introduce the following definitions:

Definition 1. Let $\varphi, \psi: M \backslash \Sigma \rightarrow H$, and let $\Sigma_{0} \subset \Sigma$. We say that $\varphi$ and $\psi$ are asymptotic near $\Sigma_{0}$ if $\operatorname{dist}(\varphi, \psi)$ is bounded in a neighborhood of 
$\Sigma_{0}$. If $M$ is non-compact, we say that $\varphi$ and $\psi$ are asymptotic at infinity if $\operatorname{dist}(\varphi, \psi) \rightarrow 0$ at infinity on $M$. If $\varphi$ and $\psi$ are asymptotic near $\Sigma$ and at infinity, we say that $\varphi$ and $\psi$ are asymptotic.

We will write $\varphi \gtrsim \psi$ to denote that $\varphi$ is asymptotic to $\psi$ near $\Sigma, \varphi \stackrel{\sim}{\sim} \psi$ to denote that $\varphi$ is asymptotic to $\psi$ at infinity, and $\varphi \sim \psi$ to denote that $\varphi$ and $\psi$ are asymptotic. These definitions are generalizations of the notion of asymptotic geodesics, which proved useful in the study of the ideal boundary of Hadamard manifolds, see [EO]. We first state the following generalization of Theorem 1 in [We2].

Theorem 1. Let $M$ be a compact Riemannian manifold of dimension $m \geqslant 2$ with $\partial M \neq \emptyset$, and let $H$ be a Hadamard manifold with pinched sectional curvatures $-b^{2} \leqslant \kappa \leqslant-a^{2}<0$. Let $\Sigma$ be a compact smooth submanifold of $M \backslash \partial M$ of co-dimension at least 2 , and assume that $\Sigma$ has finitely many connected components $\Sigma_{j}, j=1, \ldots, \ell$. For each $j=1, \ldots, \ell$, let $\varphi_{j}$ be a $\Sigma_{j}$-singular map into $\gamma_{j}$, and let $\psi: M \backslash \Sigma \rightarrow H$ be a smooth map. Then there is a unique harmonic map $\varphi: M \backslash \Sigma \rightarrow H$ such that $\varphi \stackrel{\Sigma_{j}}{\sim} \varphi_{j}$, and $\varphi=\psi$ on $\partial M$.

As mentioned earlier, $\varphi$ is smooth on $M \backslash \Sigma$. An interesting analytical question can be posed here: can more be said on the asymptotic behavior of $\varphi$ near $\Sigma$ ? To be more precise, consider a point, say $x_{0} \in \Sigma_{j}$, and let $B$ be a geodesic ball in $M$ about $x_{0}$. Let $(r, \omega)$ be a geodesic polar coordinate system on $H$ centered at the ideal boundary point $\gamma_{j}(-\infty)$, i.e.,

$$
r(p)=\lim _{t \rightarrow \infty}\left(\operatorname{dist}\left(p, \gamma_{j}(-t)\right)-t\right)
$$

is the Busemann function associated with the geodesic $\gamma_{j}(-t)[\mathrm{EO}]$, and $\omega$ is a coordinate system on the horospheres $\{r=$ const. $\}$ invariant under the geodesic vector field $\nabla r$ [We2]. It is easy to see $r \circ \varphi_{j}$ is harmonic, that $\left|r \circ \varphi-r \circ \varphi_{j}\right|$ is bounded in $B$, and that $\omega \circ \varphi$ tends to a constant on $\Sigma_{j}$. The question can then be put: how smooth are the functions $r \circ \varphi-r \circ \varphi_{j}$ and $\omega \circ \varphi$ in $B$ ? This question is also important for the application of the existence result presented next to rotating black holes. When $H=H_{\mathbb{R}}^{k}$, the real hyperbolic space, a $C^{3, \alpha}$-regularity result was proved in [LTi2].

For the application to rotating black holes, we need a version of Theorem 1 on $M=\mathbb{R}^{3}$. Consequently, we now define what we mean by singular Dirichlet data for harmonic maps with prescribed singularities on a noncompact manifold $M$.

Definition 2. Assume that $M$ is non-compact, complete, and that $\Sigma$ has finitely many connected components $\Sigma_{j}, j=1, \ldots, \ell$. A map $\psi: M \backslash \Sigma \rightarrow$ 
$H$ will be called singular Dirichlet data, if the following conditions are satisfied:

(i) $\psi \stackrel{\Sigma_{j}}{\sim} \varphi_{j}, j=1, \ldots, \ell$, for some $\Sigma_{j}$-singular maps $\varphi_{j}$ from $M \backslash \Sigma_{j}$ into geodesics $\gamma_{j}$ of $H$.

(ii) $|\tau(\psi)| \leqslant \Delta v$ for some negative bounded function $v$ on $M$ which satisfies $v(x) \rightarrow 0$ as $x \rightarrow \infty$.

Note that when $M=\mathbb{R}^{m}$ with $m \geqslant 3$, we can take $v=-\left(1+r^{2}\right)^{-\epsilon}$ in (ii), where $0<\epsilon<1 / 2$ and $r$ is the distance from the origin, to get $\Delta v \geqslant c\left(1+r^{2}\right)^{-1-\epsilon}$. Thus, in order to satisfy (ii), it suffices in this case to verify that $|\tau(\psi)|=O\left(r^{-2-\epsilon}\right)$ for some $\epsilon>0$. In the next section, we will use this fact to construct singular Dirichlet data on $\mathbb{R}^{3} \backslash \Sigma$, where $\Sigma$ is the $z$-axis with a finite number of open intervals removed.

We can now state our main result:

Theorem 2. Let $M$ be a non-compact complete Riemannian manifold of dimension $m \geqslant 3$, and let $H$ be a Hadamard manifold with pinched sectional curvatures $-b^{2} \leqslant \kappa \leqslant-a^{2}<0$. Let $\Sigma$ be a closed smooth submanifold of $M$ of co-dimension at least 2 , and assume $\Sigma$ has finitely many connected components. Let $\psi: M \backslash \Sigma \rightarrow H$ be singular Dirichlet data. Then there is a unique harmonic map $\varphi: M \backslash \Sigma \rightarrow H$ such that $\varphi \sim \psi$.

This theorem will be proved in section 3. The proof of Theorem 1 follows the same lines, and is in fact somewhat simpler. In the next section, we show how Theorem 2 is applied to obtain solutions of the Einstein/AbelianYang-Mills equations which can be interpreted as describing spacetimes containing multiple co-axially rotating black holes in equilibrium, possibly held apart by singular struts.

\section{Multiple co-axially rotating Einstein/Abelian-Yang-Mills black holes}

Consider the Einstein/Abelian-Yang-Mills equations with the Abelian gauge group $\mathbb{T}^{k}$ :

$$
\begin{gathered}
\operatorname{Ric}_{\mathbf{g}}-\frac{1}{2} R_{\mathbf{g}} \mathbf{g}=2 T_{\mathbf{F}}, \\
\mathbf{F}=d \mathbf{A}, \\
d * \mathbf{F}=0, \\
T_{\mathbf{F}}(X, Y)=\operatorname{tr}_{\mathbf{g}}\left(i_{X} \mathbf{F} \cdot i_{Y} \mathbf{F}+i_{X} * \mathbf{F} \cdot i_{Y} * \mathbf{F}\right) .
\end{gathered}
$$

Here $\mathbf{g}$ is a Lorentzian metric on a 4-dimensional manifold $\mathbf{M}, \mathrm{Ric}_{\mathbf{g}}$ is the Ricci curvature of $\mathbf{g}, R_{\mathbf{g}}$ its scalar curvature, $\mathbf{A}$ is an $\mathbb{R}^{k}$-valued one-form on $\mathbf{M}, d$ is the exterior derivative, $i_{X}$ inner multiplication by the vector 
$X, \operatorname{tr}_{\mathbf{g}}$ is the trace with respect to the metric $\mathbf{g}$, and the dot represents the standard Euclidean inner product on $\mathbb{R}^{k}$. Assume that the solution $(\mathbf{M}, \mathbf{g}, \mathbf{A})$ is stationary and axially symmetric, i.e., is invariant under an effective action of the group $G=\mathbb{R} \times S O(2)$ with timelike orbits. Assuming also that the length of the area element of the orbits has no critical points, equations (2) reduce to the axially symmetric harmonic map equations for a map $\varphi: \Omega \rightarrow H_{\mathbb{C}}^{k+1}$ from a domain $\Omega \subset \mathbb{R}^{3}$ into the complex hyperbolic space $H_{\mathbb{C}}^{k+1}$ of complex dimension $k+1$, see [We4].

We will use as a model for $H_{\mathbb{C}}^{k+1}$, the space $\mathbb{R}^{2 k+2}$ with the metric:

$$
d s^{2}=d u^{2}+e^{4 u}(d v+\boldsymbol{\chi} \cdot d \boldsymbol{\psi}-\boldsymbol{\psi} \cdot d \boldsymbol{\chi})^{2}+e^{2 u}(d \boldsymbol{\chi} \cdot d \boldsymbol{\chi}+d \boldsymbol{\psi} \cdot d \boldsymbol{\psi})
$$

where $\boldsymbol{\chi}$, and $\boldsymbol{\psi}$ are $\mathbb{R}^{k}$-valued. In these coordinates, the harmonic map equations (1) read:

$$
\begin{aligned}
\Delta u-2 e^{4 u}|\omega|^{2}-e^{2 u}(\nabla \boldsymbol{\chi} \cdot \nabla \boldsymbol{\chi}+\nabla \boldsymbol{\psi} \cdot \nabla \boldsymbol{\psi}) & =0 \\
\Delta v+2 \nabla u \nabla v+2 \omega \nabla u-2 e^{2 u} \omega(\boldsymbol{\chi} \cdot \nabla \boldsymbol{\chi}+\boldsymbol{\psi} \cdot \nabla \boldsymbol{\psi}) & =0 \\
\Delta \boldsymbol{\chi}+2 \nabla u \nabla \boldsymbol{\chi}-2 e^{2 u} \omega \nabla \boldsymbol{\psi} & =0 \\
\Delta \boldsymbol{\psi}+2 \nabla u \nabla \boldsymbol{\psi}+2 e^{2 u} \omega \nabla \boldsymbol{\chi} & =0
\end{aligned}
$$

where we have written $\omega=\nabla v+\boldsymbol{\chi} \cdot \nabla \boldsymbol{\psi}-\boldsymbol{\psi} \cdot \nabla \boldsymbol{\chi}$. Note that the left-hand sides $\left(\tau^{u}, \tau^{v}, \tau^{\chi}, \tau^{\psi}\right)$ of the equations in (3) are the components in our coordinates of the tension $\tau(\varphi)$ of any map $\varphi=(u, v, \boldsymbol{\chi}, \boldsymbol{\psi})$ not necessarily harmonic. Thus, we have:

$$
|\tau(\varphi)|^{2}=\left(\tau^{u}\right)^{2}+e^{4 u}\left(\tau^{v}+\boldsymbol{\chi} \cdot \tau^{\psi}-\boldsymbol{\psi} \cdot \tau^{\boldsymbol{\chi}}\right)^{2}+e^{2 u}\left(\left|\tau^{\boldsymbol{\chi}}\right|^{2}+\left|\tau^{\boldsymbol{\psi}}\right|^{2}\right) .
$$

Pick $I_{j}, j=1, \ldots, \ell$, bounded open intervals of the $z$-axis $\mathbb{A}$ in $\mathbb{R}^{3}$, and let $\Sigma=\mathbb{A} \backslash \bigcup_{j=1}^{\ell} I_{j}$. Denote the connected components of $\Sigma$ by $\Sigma_{j}, j=1, \ldots, \ell+1$, and let $\rho$ be the distance to the $z$-axis. It is now a straightforward matter to check, using (3) and (4), that the following lemma holds.

Lemma 1. Suppose that $\Delta u_{0}=0, u_{0} \rightarrow \infty$ on $\Sigma$, and $u_{0}+\log \rho \rightarrow$ 0 at infinity, and that $v_{0}, \chi_{0}$, and $\boldsymbol{\psi}_{0}$ are constant near each $\Sigma_{j}$, and depend only on the polar angle outside a large enough ball in $\mathbb{R}^{3}$. Let $\psi=\left(u_{0}, v_{0}, \boldsymbol{\chi}_{0}, \boldsymbol{\psi}_{0}\right): \mathbb{R}^{3} \backslash \Sigma \rightarrow H_{\mathbb{C}}^{k+1}$. Then, we have $|\tau(\psi)|=O\left(r^{-3}\right)$, and consequently $\psi$ constitutes singular Dirichlet data.

If we assume in addition a number of global hypotheses on the solution (M, g, A), namely that it is asymptotically flat, globally hyperbolic, 
that its domain of outer communications is simply connected, and furthermore, that it has an event horizon with $\ell \geqslant 1$ connected components each of which is nondegenerate, then the domain of the harmonic map $\varphi$ is precisely of the form $\mathbb{R}^{3} \backslash \Sigma$ as above, and singular boundary conditions for $\varphi$ on $\Sigma$ and at infinity are prescribed precisely by a map $\psi$ as above. Thus, the problem reduces to the following singular Dirichlet problem, see [We4].

Problem 1. Let $v_{j}$, and $\boldsymbol{\psi}_{j} \in \mathbb{R}^{k+1}, j=1, \ldots, \ell+1$, be constants, and define $\psi: \mathbb{R}^{3} \backslash \Sigma \rightarrow H_{\mathbb{C}}^{k+1}$ as in Lemma 1 so that $\psi=\left(u_{0}, v_{j}, \mathbf{0}, \boldsymbol{\psi}_{j}\right)$ in a neighborhood of $\Sigma_{j}$. Find a harmonic map $\varphi: \mathbb{R}^{3} \backslash \Sigma \rightarrow H_{\mathbb{C}}^{k+1}$ such that $\varphi \sim \psi$.

In view of Lemma 1 and Theorem 2, we obtain:

Theorem 3. Problem 1 has a unique solution.

A partial converse holds, namely, given a solution of Problem 1, one can construct a solution of equations (2) on the manifold $\mathbf{M}^{\prime}=\mathbb{R} \times\left(\mathbb{R}^{3} \backslash \mathbb{A}\right)$. In order for this manifold to be interpreted as a spacetime describing $\ell$ co-axially rotating black holes, it is necessary to extend the metric across $\Sigma$. The first step in this direction is the regularity question posed in the introduction, page 837. Once this is settled, the obstruction is a conical singularity which may appear on the axis. This singularity is easily ruled out on the unbounded components of $\Sigma$, but it is conjectured that it is always present on some bounded component. The angle deficiency of this conical singularity can be related to the force between the black holes, see [BW, We1, We3]. Thus, these spacetime should be interpreted as representing multiple co-axially rotating charged black holes, possibly held apart in equilibrium by a singular strut.

\section{Proof of Theorem 2}

The proof of Theorem 2 relies on the following simple lemma:

Lemma 2. Let $\Omega \subset M$, let $\varphi_{1}, \varphi_{2}: \Omega \rightarrow H$ be smooth maps, and let $\rho=\operatorname{dist}\left(\varphi_{1}, \varphi_{2}\right)$. Then, we have

$$
\Delta_{g}\left(\sqrt{1+\rho^{2}}\right) \geqslant-\left(\left|\tau\left(\varphi_{1}\right)\right|+\left|\tau\left(\varphi_{2}\right)\right|\right) .
$$

Proof. Let $G_{p}(q)$ denote the gradient of the function $q \mapsto \operatorname{dist}(p, q)$ on $H$ evaluated at $q \neq p$, and let $L$ denote the Hessian of the function $(p, q) \mapsto$ $\operatorname{dist}(p, q)$ on $H \times H$ as a quadratic form on $T(H \times H)$ away from the 
diagonal $\{(p, p) \in H \times H\}$. The proof of the lemma relies on the following identity:

$$
\begin{aligned}
\Delta_{g} \rho=h\left(G_{\varphi_{1}}\left(\varphi_{2}\right), \tau\left(\varphi_{2}\right)\right)+h\left(G_{\varphi_{2}}\left(\varphi_{1}\right)\right. & \left., \tau\left(\varphi_{1}\right)\right) \\
& +L\left(d \varphi_{1}+d \varphi_{2}, d \varphi_{1}+d \varphi_{2}\right),
\end{aligned}
$$

which holds wherever $\rho>0$. Since $\left|G_{p}(q)\right|=1$ for all $q \neq p$, and since $L \geqslant 0$, due to the negative curvature of $H$ [SY, p. 368], this implies that $\Delta_{g} \rho \geqslant-\left(\left|\tau\left(\varphi_{1}\right)\right|+\left|\tau\left(\varphi_{2}\right)\right|\right)$ whenever $\rho>0$, hence also

$$
\Delta_{g}\left(\sqrt{1+\rho^{2}}\right)=\frac{\rho\left(1+\rho^{2}\right) \Delta_{g} \rho+|\nabla \rho|^{2}}{\left(1+\rho^{2}\right)^{3 / 2}} \geqslant-\left(\left|\tau\left(\varphi_{1}\right)\right|+\left|\tau\left(\varphi_{2}\right)\right|\right),
$$

whenever $\rho>0$. However, the function $\sqrt{1+\rho^{2}}$ has a global minimum on $\{\rho=0\}$, hence its Hessian, and consequently also its Laplacian are non-negative there.

We first establish the uniqueness statement in Theorem 2. If $\varphi_{1}, \varphi_{2}: M \backslash$ $\Sigma \rightarrow H$ are both harmonic maps asymptotic to $\psi$, then $\varphi_{1} \sim \varphi_{2}$, and from Lemma 2, we have that $\operatorname{dist}\left(\varphi_{1}, \varphi_{2}\right)$ is a bounded subharmonic function on $M \backslash \Sigma$ and tends to 0 at infinity. It is not difficult to see that, since $\Sigma$ has codimension at least 2 , the function $\operatorname{dist}\left(\varphi_{1}, \varphi_{2}\right)$ is then weakly subharmonic on $M$, cf. [We2, Lemma 8]. Hence, by the maximum principle, it vanishes on $M$.

Fix $\delta>0$, and let $M_{\delta}=B_{1 / \delta}\left(x_{0}\right) \bigcap\{x \in M: \operatorname{dist}(x, \Sigma)>\delta\}$. Then $\overline{M_{\delta}}$ is compact and $\psi$ is smooth on $M_{\delta}$, hence, since $H$ is negatively curved, there is a smooth harmonic map $\varphi_{\delta}: M_{\delta} \rightarrow H$ such that $\varphi_{\delta}=\psi$ on $\partial M_{\delta}$. Let $\rho=\operatorname{dist}\left(\varphi_{\delta}, \psi\right)$ and note that by Lemma 2, and (ii) in Definition 2, we have:

$$
\Delta_{g}\left(v+\sqrt{1+\rho^{2}}\right) \geqslant 0
$$

on $M_{\delta}$, for some function $v<0$ on $M_{\delta}$, such that $v(x) \rightarrow 0$ as $x \rightarrow \infty$. We have $v+\sqrt{1+\rho^{2}} \leqslant 1$ on $\partial M_{\delta}$, hence by the maximum principle, we obtain:

$$
\operatorname{dist}\left(\varphi_{\delta}, \psi\right) \leqslant \sqrt{v(v-2)} .
$$

In particular, $\operatorname{dist}\left(\varphi_{\delta}, \psi\right) \leqslant C$ for some constant $\mathrm{C}$ independent of $\delta$.

Fix now $\delta_{0}>0$, and for $0<\delta<\delta_{0}$, consider $\varphi_{\delta}$ restricted to $M_{\delta_{0}}$. This is a family of smooth harmonic maps into a fixed ball $B_{R} \subset H$. Indeed, $\psi\left(M_{\delta_{0}}\right)$ is bounded, and $\varphi_{\delta}\left(M_{\delta_{0}}\right)$ lies within distance $C$ of this set. This implies that one can pick a subsequence $\varphi_{i}=\varphi_{\delta_{i}}$ with $\delta_{i} \rightarrow 0$ such that $\varphi_{i}$ converges together with two derivatives, uniformly on compact subsets of $M_{\delta_{0}}$, to a harmonic map $\psi_{\delta_{0}}: M_{\delta_{0}} \rightarrow H$. To see this, it suffices to 
establish, on each $\Omega \subset \subset M_{\delta_{0}}$, uniform bounds on $\varphi_{\delta}$ in $C^{2, \alpha}$ for some $\alpha>0$.

This is quite standard, but for the sake of completeness, we demonstrate here how this is done. Pick a point $p_{0} \in H \backslash B_{R}$, and introduce geodesic polar coordinates $(r, \omega)$ at $p_{0}$. In these coordinates, the metric $h$ of $H$ reads:

$$
d s^{2}=d r^{2}+Q(d \omega, d \omega)
$$

We view $Q$ as a function on $H \backslash\left\{p_{0}\right\}$ with values in the quadratic forms on $\mathbb{R}^{n-1}$. As such, due to the negative curvature of $H, Q$ is increasing along the outgoing geodesics from $p_{0}$. In fact, we have:

$$
2 a Q(X, X) \leqslant \frac{\partial}{\partial r}(Q(X, X)) \leqslant 2 b Q(X, X),
$$

for every $X \in R^{n-1}$. The energy density of a map $\varphi$ into $H \backslash\left\{p_{0}\right\}$ is given by:

$$
|d \varphi|^{2}=|\nabla(r \circ \varphi)|^{2}+Q(\nabla(\omega \circ \varphi), \nabla(\omega \circ \varphi)) .
$$

Here, we wrote

$$
Q(\nabla(\omega \circ \varphi), \nabla(\omega \circ \varphi))=\sum_{\mu=1}^{m} Q\left(\nabla_{X_{\mu}}(\omega \circ \varphi), \nabla_{X_{\mu}}(\omega \circ \varphi)\right),
$$

where $\left\{X_{1}, \ldots, X_{m}\right\}$ is a local orthonormal frame on $M$.

To simplify the notation, we omit the subscript $\delta$, and write $\varphi=(r, \omega)$. Thus, we assume that $\varphi=(r, \omega): M_{\delta_{0}} \rightarrow B_{R} \subset H$ is a harmonic map. We first show that if $\Omega \subset \subset M_{\delta_{0}}$, then $E_{\Omega}(\varphi) \leqslant C$, where $C$ depends only on $\Omega$, and the radius of $B$. To this end, note that, in view of (8), we have:

$$
\Delta_{g} r=\frac{\partial}{\partial r}(Q(\nabla \omega, \nabla \omega)) \geqslant 2 a Q(\nabla \omega, \nabla \omega) \geqslant 0 .
$$

Let $\chi \in C_{0}^{\infty}\left(M_{\delta_{0}}\right)$ be a smooth cut-off function for $\Omega$, i.e., $0 \leqslant \chi \leqslant 1$, and $\chi=1$ on $\Omega$. Multiply (9) by $\chi^{2} r$, and integrate over $M_{\delta_{0}}$. We obtain, after integrating by parts:

$$
\begin{aligned}
\int_{M_{\delta_{0}}} \chi^{2}|\nabla r|^{2} & \leqslant 2 \int_{M_{\delta_{0}}} \chi r g(\nabla r, \nabla \chi) \\
& \leqslant 2 \sup _{M_{\delta_{0}}} r\left(\int_{M_{\delta_{0}}} \chi^{2}|\nabla r|^{2}\right)^{1 / 2}\left(\int_{M_{\delta_{0}}}|\nabla \chi|^{2}\right)^{1 / 2}
\end{aligned}
$$

Dividing by $\left(\int_{M_{\delta_{0}}} \chi^{2}|\nabla r|^{2}\right)^{1 / 2}$, and squaring, we see that:

$$
\int_{M_{\delta_{0}}} \chi^{2}|\nabla r|^{2} \leqslant 4 \sup _{M_{\delta_{0}}}\left(r^{2}\right) \int_{M_{\delta_{0}}}|\nabla \chi|^{2}
$$


Now, multiply (9) by $\chi^{2}$, and integrate by parts:

$$
\int_{M_{\delta_{0}}} \chi^{2} Q(\nabla \omega, \nabla \omega) \leqslant \frac{1}{a} \int_{M_{\delta_{0}}} \chi \nabla \chi \cdot \nabla r \leqslant \frac{2}{a} \sup _{M_{\delta_{0}}} r \int_{M_{\delta_{0}}}|\nabla \chi|^{2} .
$$

Combining (10) and (11), we conclude as claimed that:

$$
E_{\Omega}(\varphi) \leqslant\left(4 \sup _{M_{\delta_{0}}} r^{2}+\frac{2}{a} \sup _{M_{\delta_{0}}} r\right) \int_{M_{\delta_{0}}}|\nabla \chi|^{2} .
$$

Next, we use the Bochner identity:

$$
\Delta_{g}\left(|d \varphi|^{2}\right)=|\nabla d \varphi|^{2}+{ }^{M} \operatorname{Ric}(d \varphi, d \varphi)-{ }^{H} \operatorname{Riem}(d \varphi, d \varphi, d \varphi, d \varphi)
$$

to obtain a pointwise bound on $|d \varphi|^{2}$ which depend only on $\Omega$. Here, ${ }^{M}$ Ric denotes the Ricci curvature of the metric $g$ on $M,{ }^{H}$ Riem denotes the Riemann curvature of the metric $h$ on $H$, and we have written:

$$
\begin{gathered}
{ }^{M} \operatorname{Ric}(d \varphi, d \varphi)=\sum_{\mu=1}^{m}{ }^{M} \operatorname{Ric}\left(X_{\mu}, X_{\mu}\right) h\left(\nabla_{X_{\mu}} \varphi, \nabla_{X_{\mu}} \varphi\right), \\
{ }^{H} \operatorname{Riem}(d \varphi, d \varphi, d \varphi, d \varphi)=\sum_{\mu, \nu=1}^{m}{ }^{H} \operatorname{Riem}\left(\nabla_{X_{\mu}} \varphi, \nabla_{X_{\nu}} \varphi, \nabla_{X_{\mu}} \varphi, \nabla_{X_{\nu}} \varphi\right),
\end{gathered}
$$

where $\left\{X_{1}, \ldots, X_{m}\right\}$ is a local orthonormal frame on $M$. Due to the negative curvature of $H$, and to the fact that $M_{\delta_{0}}$ is bounded, we obtain

$$
\Delta_{g}\left(|d \varphi|^{2}\right) \geqslant-\left(\left.\sup _{M_{\delta_{0}}}\right|^{M} \operatorname{Ric} \mid\right)|d \varphi|^{2} .
$$

Standard elliptic theory now implies that

$$
\sup _{\Omega^{\prime}}|d \varphi|^{2} \leqslant C^{\prime} \int_{\Omega}|d \varphi|^{2} \leqslant C^{\prime \prime}
$$

where the constants $C^{\prime}$ and $C^{\prime \prime}$ depend only on $\Omega$. Finally, we substitute this estimate into the right-hand side of (1), to deduce, again from standard elliptic theory, a uniform bound on $\varphi$ in the Sobolev space $W^{2, p}$ for any $p>1$. If we take $p>m$, this implies a uniform bound on $\varphi$ in the Hölder space $C^{1, \alpha}$, for $\alpha=1-m / p$. Again, substitute in the right-hand side to get a uniform bound on $\varphi$ in $C^{2, \alpha}$. This can be iterated to get uniform bounds on any number of derivatives of $\varphi$. We should point out that at each step the estimate requires that we take the norms on a smaller subset $\Omega_{k} \subset \subset \Omega_{k-1}$, but it can easily be arranged that the last one of these is $\Omega$. 
By taking a sequence $M_{\delta_{i}}$ exhausting $M$, and by passing to a diagonal subsequence, we obtain a sequence of harmonic maps $\varphi_{i}: M_{\delta_{i}} \rightarrow H$ such that (7) holds, and such that $\varphi_{i}$ converges, uniformly on each set $\Omega \subset \subset M$, to a harmonic map $\varphi$. It remains to show that $\varphi \sim \psi$. Since $\varphi_{i} \rightarrow \varphi$ pointwise, we have from (7) that $\varphi$ also satisfies:

$$
\operatorname{dist}(\varphi, \psi) \leqslant \sqrt{v(v-2)},
$$

which immediately implies $\varphi \underset{\sim}{\sim}$, and also $\varphi \underset{\sim}{\sim}$ since $v(x) \rightarrow 0$ as $x \rightarrow$ $\infty$ in $M$. This completes the proof of Theorem 2 .

The proof of Theorem 1 is somewhat simpler. It suffices to note that one can assume without loss of generality that $\psi=\varphi_{j}$ in a neighborhood of $\Sigma_{j}$. We then have $|\tau(\psi)| \leqslant C$, and the proof now proceeds as before with $M_{\delta}=\{x \in M: \operatorname{dist}(x, \Sigma)>\delta\}$, without any need for (ii) in Definition 2 .

\section{Acknowledgment}

We wish to thank Piotr Chrusciel for valuable discussions concerning the problem addressed in this paper.

\section{References}

[BW] R. Bach and H. Weyl, Neue Lösungen der Einsteinschen Gravitationsgleichungen, Math. Z. 13 (1921), 132-145.

[EO] P. Eberlein and B. O'Neill, Visibility manifolds, Pacific J. Math 46 (1973), 45-109.

[ES] J. Eells and J. H. Sampson, Harmonic mappings of Riemannian manifolds. Amer. J. Math. 86 (1964), 109-164.

[H] R. Hamilton, Harmonic maps of manifolds with boundary, Lecture Notes in Mathematics No. 471, Springer-Verlag, Berlin, Heidelberg, New York, 1975.

[LTi2] Y. Li and G. Tian, Regularity of harmonic maps with prescribed asymptotic behavior and applications, Comm. Math. Phys. 149 (1992), No. 1, 1-30.

[SU1] R. Schoen and K. Uhlenbeck, A regularity theory for harmonic maps, J. Diff. Geom. 17 (1982), 307-335.

[SU2] _ Boundary regularity and the Dirichlet problem for harmonic maps, J. Diff. Geom. 18 (1983), 253-268.

[SY] R. Schoen and S. T. Yau, Compact group actions and the topology of manifolds with non-positive curvature, Topology 18 (1979), 361-380.

[We1] G. Weinstein, On the force between rotating co-axial black holes, Trans. Amer. Math. Soc. 343 (1994), 899-906.

[We2] _ On the Dirichlet problem for harmonic maps with prescribed singularities, Duke Math. J. 77 (1995) 135-165.

[We3] , N-black hole stationary and axially symmetric solutions of the Einstein-Maxwell equations, Comm. Partial Diff. Eq. 21 (1996) 1389-1430.

[We4] _ Harmonic maps with prescribed singularities on unbounded domains, Amer. J. Math., 118 (1996) 689-700.

Department of Mathematics, University of Alabama at Birmingham

E-mail address: weinstei@math.uab.edu 